\title{
China and Multiomics Research and Development Ecosystem: A Horizon Scanning and Current State of the Art
}

\author{
Biaoyang $\operatorname{Lin}^{1-3}$
}

\section{Introduction}

$\mathbf{L}$ ONG GONE ARE THE DAYS when systems sciences relied primarily on genomics. Multiomics research and development $(\mathrm{R} \& \mathrm{D})$ encompassing not only genomics but also metabolomics, metagenomics, glycomics, and other omics, not to mention their applications to human diseases, ecology, and health interventions (drugs, nutrition, vaccines, etc.) have resulted in a kaleidoscope of novel scientific fields in integrative biology and system sciences. This is being reflected in both public and private sectors as well. Studying the hitherto underappreciated linkages between emerging technologies and societal change is another key pillar of multiomics R\&D.

Many countries are both striving and struggling to cultivate multiomics R\&D as a key driver of life sciences innovation in the early 21 st century. China is no exception in this context and has made important strides in multiomics R\&D for the past decade.

The multiomics R\&D began to gain momentum in China with the initiative of precision medicine announced in 2016. Between 2016 and 2020, the Ministry of Science and Technology (MOST) made major efforts to support research and researchers in precision medicine building capacity in multiomics R\&D, large sample sized cohort studies, big data storage and sharing, data integration and utilization, among others.

As we shift our gaze toward the new year 2020, I highlight what I consider, in my personal opinion, to be three actionable emerging challenges in the field of multiomics and precision medicine $\mathrm{R} \& \mathrm{D}$, specifically in China that can transform to prospects for clinical, translational, ecological, and/or discovery life sciences innovation internationally.

\section{Actionable Challenge 1}

\section{Creating a nationwide patient registry}

There is a lack of nationwide patient registry in China for effective analysis of multiomics data for clinical implications. For example, China has the largest hepatitis B virus-infected population and accounts for $>50 \%$ of the hepatocellular carcinoma worldwide (Bray et al., 2018; McGlynn et al., 2015). But milestone initiatives such as the "Risk of Hepatocellular Carcinoma in Patients Treated with Entecavir vs Tenofovir for Chronic Hepatitis B: A Korean Nationwide Cohort Study" (Choi et al., 2019) are rather difficult for Chinese scientists currently because of the lack of a nationwide patient registry.

Unless we create a nationwide patient registry in China with the help of the national health insurance program and the government, the advantage of the large population size for precision medicine research in the country cannot be fully realized. Without registries and documentation to enable rich phenotypic data sets, the promises of Big Data can be hollowed out. Still, with collective action to be harnessed by the government and the national health insurers, this challenge remains to be addressed and transformed to an advantage building on the large population size in China.

\section{Actionable Challenge 2}

\section{Governance innovation to cultivate responsible and veritable innovations}

True innovative multiomics $R \& D$ requires large and longterm investment and good governance. The current Chinese society seems to award quick and short-term gains. In fact, immediacy and short-term vision are problematic in various geographies and institutions. The values that drive science and collaboration ought to be examined so as to cultivate enduring scientific investments that can benefit many in China and globally. For example, there are enormous pressures on academics and physicians for rapid research outputs, publications, and results. Although being "results-oriented" is valuable, this should not come in the expense of a loss in long-term vision in science and scientific impacts.

Intellectual property issues, too, can be facilitators or barriers to investments in science and technology-based innovation in China if and when products are copied or imitated from other countries. Negotiating the price for innovative drugs and/or the long wait for the price approval for novel medical devices and health care services often hinder the incentives and recouping the costs of the R\&D. In all, these examples attest to a need for governance innovation in ways that build on a deeper understanding of the human and institutional values underlying progress in science, technology, and society. Critically informed good governance is often overlooked by scientists but remains a crucial pillar of responsible and veritable innovation in health, engineering, and

\footnotetext{
${ }^{1}$ Systems Biology Center, Zhejiang-California International Nanosystems Institute (ZCNI), Zhejiang University, Hangzhou, China.

${ }^{2}$ Collaborative Innovation Center for Diagnosis and Treatment of Infectious Diseases, The First Affiliated Hospital, School of Medicine, Zhejiang University, Hangzhou, China.

${ }^{3}$ University of Washington School of Medicine, Seattle, Washington.
} 
integrative biology, not to mention of science policy in the 21 st century.

\section{Actionable Challenge 3}

Contributing to international standards and guidelines and cultivating global collaborations

Participating and contributing to setting of international standards and guidelines, and, in particular, standards in clinical practice guidelines informed by multiomics and precision medicine R\&D are crucial for success in the multiomics field. Although China has the largest patient population in many diseases, China's contribution to and its recognition in the international standards and clinical practice guidelines are mostly lacking or very limited.

Oftentimes, Chinese scientists lack the confidence or means to advocate for policy research that might help setting guidelines. Most of the clinical practice guidelines in China were adopted from the guidelines in North America or Europe, which do not always address the local needs of the scientists and patients in China. A related challenge is to cultivate and sustain scientific collaborations between China and the United States, although a trade war and tensions are escalating between the countries. Trust ought to be rebuilt to maintain and further promote scientific collaborations between the two countries that continue to make significant contributions to the global science and technology innovation ecosystem.

\section{Conclusions}

China has invested heavily in multiomics R\&D and precision medicine but has yet to realize landmark breakthrough contributions in the field and needs to be better recognized in international standards and clinical practice guidelines. By addressing the aforementioned three actionable challenges, China would be well poised to make important strides and impacts in multiomics and precision medicine, mirroring and matching its strong economic success trajectory in the past half century.

\section{Disclaimer}

The views expressed are the personal opinions of the author only.

\section{Disclosure Statement}

The author declares there are no conflicting financial interests.

\section{Funding Information}

No funding was received for this article.

\section{References}

Bray F, Ferlay J, Soerjomataram I, Siegel RL, Torre LA, and Jemal A. (2018). Global cancer statistics 2018: GLOBOCAN estimates of incidence and mortality worldwide for 36 cancers in 185 countries. CA Cancer J Clin 68, 394-424.

Choi J, Kim HJ, Lee J, Cho S, Ko MJ, and Lim YS. (2019). Risk of hepatocellular carcinoma in patients treated with entecavir vs tenofovir for chronic hepatitis B: A Korean Nationwide Cohort Study. JAMA Oncol 5, 30-36.

McGlynn KA, Petrick JL, and London WT. (2015). Global epidemiology of hepatocellular carcinoma: An emphasis on demographic and regional variability. Clin Liver Dis 19, 223238.
Address correspondence to: Biaoyang Lin, PhD

Systems Biology Center Zhejiang-California International Nanosystems Institute (ZCNI) Zhejiang University Hangzhou 310058 China E-mail: biaoylin@gmail.com 